\title{
Best Approximation of Positive Power Series
}

\author{
By B. L. R. Shawyer*
}

\begin{abstract}
This paper extends work of Fiedler, Jurkat and the present author to series of the form $\sum a_{n} x^{n}$ where $\left\{a_{n}\right\}$ is a moment sequence and $0<x<1$. In the cases where it is possible to calculate it exactly, we find the best $L^{p}$ approximation to the sum of the series and the actual terms of the matrices involved. We have an advantage over accelerators commonly used for accelerating convergence in that we know explicitly the errors in our calculations.
\end{abstract}

1. Introduction. In recent papers, Jurkat and Shawyer [3], Fiedler and Jurkat [2] and Shawyer [4] have considered the problem of the best approximation to the sum of convergent series of the form $\sum(-1)^{n} a_{n} x^{n}$ with $0<x \leqslant 1$, obtained from the first $(n+1)$ terms of the series, where $\left\{a_{n}\right\}$ is a moment sequence with

$$
a_{n}=\int_{0}^{1} t^{n} d \phi \quad \text { and } \quad \int_{0}^{1}|d \phi|=1 .
$$

In this paper, we consider series of the form $\sum a_{n} x^{n}$ with $0<x<1$, where $a_{n}$ is as above. The results obtained here are similar to those in [4].

We show the best approximation explicitly for the cases in which it can be calculated. In particular, in the notation of [4], we show that the error of the best $L^{\infty}$ approximation is given by

$$
\varepsilon_{n}^{(\infty)}(B)=\frac{x}{2(1-x)}\left(\frac{2}{x}-1-\frac{2}{x} \sqrt{1-x}\right)^{n} .
$$

From this we observe that it is not appropriate to let $x \rightarrow 1$ - since the factor $x / 2(1-x)$ tends to infinity and $(2 / x-1-2 \sqrt{1-x} / x)$ tends to 1 .

The matrices obtained are not regular on the class of convergent series but are regular on the subclass considered.

Let $C=\left(c_{n, k}\right)$ be a series-to-sequence triangular matrix, so that $c_{n, k}=0$ whenever $k>n$. Define, for $0<x<1$,

$$
\sigma_{n}=\sum_{k=0}^{n} c_{n, k} a_{k} x^{k} \quad \text { and } \quad \gamma_{n}(t)=\sum_{k=0}^{n} c_{n, k} t^{k} .
$$

Thus

$$
\sigma_{n}=\int_{0}^{1} \sum_{k=0}^{n} c_{n, k}(x t)^{k} d \phi(t)=\int_{0}^{1} \gamma_{n}(x t) d \phi
$$

Received March 28, 1983; revised January 30, 1984.

1980 Mathematics Subject Classification. Primary 41A25, 41A50; Secondary 41A10, 40A25.

* Supported in part by the Natural Sciences and Engineering Research Council of Canada. 
Also

$$
\sum_{k=0}^{n} a_{k} x^{k}=\int_{0}^{1} \sum_{k=0}^{n}(x t)^{k} d \phi(t)=\int_{0}^{1} \frac{d \phi(t)}{1-x t}+r_{n}
$$

where

$$
r_{n}=-x^{n+1} \int_{0}^{1} \frac{t^{n+1}}{1-x t} d \phi(t) \rightarrow 0 \quad \text { as } n \rightarrow \infty
$$

Define

$$
s(x)=\int_{0}^{1} \frac{d \phi(t)}{1-x t}
$$

so that

$$
\sigma_{n}-s(x)=\int_{0}^{1}\left(\gamma_{n}(x t)-\frac{1}{1-x t}\right) d \phi(t)
$$

and so

$$
\left|\sigma_{n}-s(x)\right| \leqslant \max _{0 \leqslant t \leqslant 1}\left|\gamma_{n}(x t)-\frac{1}{1-x t}\right| \int_{0}^{1}|d \phi| .
$$

Considering without loss of generality, the class in which $\int_{0}^{1}|\phi(t)| d t=1$ and taking the supremum over this class, we obtain that

$$
\sup \left|\sigma_{n}-s(x)\right|=\max _{0 \leqslant t \leqslant 1}\left|\gamma_{n}(x t)-\frac{1}{1-x t}\right|=\left\|\gamma_{n}(x t)-\frac{1}{1-x t}\right\|_{\infty} .
$$

For the subclasses given by $\int_{0}^{1}|\psi(t)|^{q} d t=1(1 / p+1 / q=1)$ we obtain that

$$
\sup \left|\sigma_{n}-s(x)\right|=\left(\int_{0}^{1}\left|\gamma_{n}(x t)-\frac{1}{1-x t}\right|^{p} d t\right)^{1 / p}=\left\|\gamma_{n}(x t)-\frac{1}{1-x t}\right\|_{p} .
$$

Thus we define the error for the row of order $n$ of the matrix $C$, operating on the first $(n+1)$ terms of the series in our class to be

$$
\varepsilon_{n}^{(p)}(C)=\left\|\gamma_{n}(x t)-\frac{1}{1-x t}\right\|_{p} \quad(1 \leqslant p \leqslant \infty)
$$

where the norm is taken with respect to $t$ over the interval $[0,1]$. For each $n$, the best approximation occurs when this error is minimal. So we let $\gamma_{n}$ vary over the class of polynomials of degree $n$.

As in [3], let $B=\left(b_{n, k}\right)$ be the corresponding sequence-to-sequence matrix and $\beta_{n}(t)$, its row polynomial, so that

$$
t \beta_{n}(t)=\gamma_{n}(0)+(1-t) \gamma_{n}(t)
$$

2. Solution to the Problem When $p=\infty$. To find the best approximation, we must minimize the error

$$
\varepsilon_{n}^{(\infty)}(C)=\left\|\gamma_{n}(x t)-\frac{1}{1-x t}\right\|_{\infty}=\max _{-1 \leqslant v \leqslant 1}\left|\gamma_{n}\left(\frac{x}{2}(1-v)\right)-\frac{-2 / x}{v-(2 / x-1)}\right| .
$$

We must therefore apply Čebyšev's lemma (Lemma 2.1 in [3]) with $a=2 / x-1$ to find the corresponding best polynomial, $p_{n}(t)$ approximation to $1 /(v-a)$. We then set $t=2 a / x-1$ and multiply by $-2 / x$. 
The number $\alpha$ in Čebyšev's lemma is taken then

$$
\alpha=\alpha(x)=\frac{2}{x}-1-\sqrt{\left(\frac{2}{x}-1\right)^{2}-1}=\frac{2-x-2 \sqrt{1-x}}{x}<1 \text { for } 0<x<1 .
$$

Let $\alpha(1)=1$ and $\alpha(0)=0$ (by continuity). It is easy to see that $\alpha(x) \sim x / 4$ as $x \rightarrow 0+$. We then obtain that

$$
\varepsilon_{n}^{(\infty)}(C)=\frac{2}{x} \cdot \frac{\{\alpha(x)\}^{n}}{a^{2}-1}=\frac{x}{2(1-x)}\{\alpha(x)\}^{n}
$$

We now follow the development as in [4] and [3] to obtain $\gamma_{0}(u)=x / 2(1-x)$ and, for $n>0$,

where

$$
\gamma_{n}(u)=\frac{1}{u-1}\left\{\frac{x^{2}\{\alpha(x)\}^{n}}{8(1-x)} W_{n+1}\left(\frac{2 u}{x}-1\right)-1\right\},
$$

$$
W_{n+1}(t)=\frac{1}{\alpha(x)} T_{n+1}(t)-2 T_{n}(t)+\alpha(x) T_{n-1}(t)
$$

and $T_{n}(t)$ is the Čebyšev polynomial of order $n$. Now

$$
\begin{aligned}
W_{n+1}(-1) & =\frac{1}{\alpha(x)} T_{n+1}(-1)-2 T_{n}(-1)+\alpha(x) T_{n-1}(-1) \\
& =(-1)^{n+1}\left\{\frac{1}{\alpha(x)}+2+\alpha(x)\right\},
\end{aligned}
$$

so that

$$
\gamma_{n}(0)=1-\frac{\{\alpha(x)\}^{n} x^{2} W_{n+1}(-1)}{8(1-x)}=1+\frac{(-1)^{n}\{\alpha(x)\}^{n} x^{2}}{2(1-x)}
$$

$\rightarrow 1$ as $n \rightarrow \infty$ for each $x \in(0,1)$.

Noting that

$$
T_{n}(-u)=(-1)^{n} T_{n}(u) \text { and } T_{n}(1+2 v)=\sum_{k=0}^{n} \frac{n(n+k-1) !}{(n-k) ! 2 k !}(4 v)^{k}
$$

(see [3]), we obtain

$$
T_{n}\left(\frac{2 u}{x}-1\right)=(-1)^{n} \sum_{k=0}^{n} \frac{n(n+k-1) !}{(n-k) ! 2 k !}\left(-\frac{4}{x}\right)^{k} u^{k},
$$

so that $W_{n+1}(2 u / x-1)$ can be obtained explicitly as a polynomial in $u$. Using this and $t \beta_{n}(t)=\gamma_{n}(0)+(1-t) \gamma_{n}(t)$, we obtain that for $0 \leqslant k \leqslant n$

$$
\begin{aligned}
b_{n, k}^{(\infty)}= & \frac{\{\alpha(x)\}^{n} x^{2}(-1)^{n+k} 4^{k+1}}{8(1-x) x^{k+1}}\left\{\frac{(n+k-1) !}{(2 k+2) !(n-k) !}\right\} \\
& \cdot\left\{\frac{(n+1)(n+1+k)(n+k)}{\alpha(x)}+2 n\left(n^{2}-k^{2}\right)\right. \\
+\alpha(x)(n-1)(n-1-k)(n-k)\} & \\
= & \frac{\{\alpha(x)\}^{n}(-1)^{n+k} 2^{2 k-1}(n+k-1) !}{(1-x) x^{k-1}(2 k+2) !(n-k) !} \alpha_{n, k}^{(\infty)},
\end{aligned}
$$


say, where

$$
\begin{aligned}
\alpha_{n, k}^{(\infty)}= & \frac{(n+1)}{\alpha(x)}\left\{k^{2}+k(2 n+1)+n(n+1)\right\}+2 n\left(n^{2}+k^{2}\right) \\
& +\alpha(x)(n-1)\left\{k^{2}-k(2 n-1)+n(n-1)\right\} \\
= & k^{2}\left\{n\left(\frac{1}{\alpha(x)}-2+\alpha(x)\right)+\left(\frac{1}{\alpha(x)}-\alpha(x)\right)\right\} \\
& +k\left\{\left(2 n^{2}-1\right)\left(\frac{1}{\alpha(x)}-\alpha(x)\right)+3 n\left(\frac{1}{\alpha(x)}+\alpha(x)\right)\right\} \\
& +n\left\{\frac{(n+1)^{2}}{\alpha(x)}+2 n^{2}+\alpha(x)(u-1)^{2}\right\}>0
\end{aligned}
$$

since

$$
\frac{1}{\alpha(x)}-\alpha(x)>0 \text { and } \frac{1}{\alpha(x)}-2+\alpha(x)=\left(\frac{1}{\sqrt{\alpha(x)}}-\sqrt{\alpha(x)}\right)^{2}>0 .
$$

Thus $b_{n, k}^{(\infty)}=(-1)^{n+k} \beta_{n, k}^{(\infty)}$, say, where $\beta_{n, k}^{(\infty)}>0$.

We have already noted that

$$
\sum_{k=0}^{n} b_{n, k}^{(\infty)}=\gamma_{n}(0) \rightarrow 1 \quad \text { as } n \rightarrow \infty
$$

It is easy to show that

$$
\lim _{n \rightarrow \infty} b_{n, k}^{(\infty)}=0 \quad \text { for each } k \text { and for each } x .
$$

We thus have the row sum and column limit conditions of Toeplitz's Theorem. However we shall see below that the row norm condition is not satisfied. We thus have a positive nonregular triangular matrix. It is, however, regular for our class.

To see this, we observe that

$$
\begin{aligned}
\sum_{k=0}^{n}\left|b_{n, k}^{(\infty)}\right|= & \sum_{k=0}^{n} \frac{\{\alpha(x)\}^{n} 2^{2 k-1}(n+k-1) !}{(1-x) x^{k-1}(2 k+2) !(n-k) !} \alpha_{n, k}^{(\infty)} \\
=\frac{\{\alpha(x)\}^{n} x^{2}}{8(1-x)}\left[\frac{T_{n+1}(1+2 / x)}{\alpha(x)}\right. & \\
& \left.+2 T_{n}\left(1+\frac{2}{x}\right)+\alpha(x) T_{n-1}\left(1+\frac{2}{x}\right)-\frac{4}{x}\right] .
\end{aligned}
$$

(This is readily obtained by starting with the right-hand side and proceeding as with the treatment above.) From $T_{n}(u)=\frac{1}{2}\left\{\left(u-\sqrt{u^{2}-1}\right)^{n}+\left(u+\sqrt{u^{2}-1}\right)^{n}\right\}$ we obtain that

$$
T_{n}\left(1+\frac{2}{x}\right)=\frac{1}{2}\left\{\{\lambda(x)\}^{n}+\{\lambda(x)\}^{-n}\right\}
$$

where $\lambda(x)=(2+x-2 \sqrt{1+x}) / x$. It is easy to see that $\alpha(x)>\lambda(x)>0$ for $0<x<1$ so that $\{\alpha(x) / \lambda(x)\}^{n} \rightarrow \infty$ as $n \rightarrow \infty$ for each $x \in(0,1)$. Hence $\sum_{k=0}^{n}\left|b_{n, k}^{(\infty)}\right| \rightarrow \infty$ as $n \rightarrow \infty$ for each $x \in(0,1)$.

3. Solution to the Problem When $p=1$. It is possible to solve this problem explicitly when $p=2$ using the techniques developed in [4]. For the sake of brevity, 
we shall omit this here and merely state that the corresponding best matrix $B^{(2)}=\left(b_{n, k}^{(2)}\right)$ has similar properties to the matrix $B^{(\infty)}$ found above and the matrix $B^{(1)}$ found below.

In the case of $p=1$, we follow the procedure of Section 4 of [4] and obtain that the error in the approximation is given by

$$
\begin{aligned}
\varepsilon_{n}^{(1)}(C) & =\left\|\gamma_{n}(x u)-\frac{1}{1-x u}\right\|_{1}=\int_{0}^{1}\left|\gamma_{n}(x u)-\frac{1}{1-x u}\right| d u \\
& =\frac{1}{x} \int_{1}^{1}\left|-\frac{x}{2} \gamma_{n}\left(\frac{x(1+v)}{2}\right)-\frac{1}{v-(2 / x-1)}\right| d v \\
& =\frac{1}{x} \int_{-1}^{1}\left|p_{n}(v)-\frac{1}{v-a}\right| d v, \text { say, }
\end{aligned}
$$

where $a=2 / x-1>1$.

Applying Markov's Theorem [1, p. 82] as in [4], we obtain that

$$
\varepsilon_{n}^{(1)}(C)=\frac{2}{x} \log \frac{1+\{\alpha(x)\}^{n+2}}{1-\{\alpha(x)\}^{n+2}} .
$$

It is easy to show that, for $t>0$,

$$
\log \left(1+\frac{1}{t}\right)<\frac{1}{t}+\frac{1}{t+1}-\frac{1}{t+\frac{1}{2}}
$$

We use this inequality with $t=\left(1-\{\alpha(x)\}^{n+2}\right) / 2\{\alpha(x)\}^{n+2}$ and obtain

$$
\varepsilon_{n}^{(1)}(C)<\frac{4}{x}\{\alpha(x)\}^{n+2}\left(\frac{1+\{\alpha(x)\}^{2 n+4}}{1-\{\alpha(x)\}^{2 n+4}}\right) \leqslant \frac{4}{x}\{\alpha(x)\}^{n+2}\left(\frac{1+\{\alpha(x)\}^{4}}{1-\{\alpha(x)\}^{4}}\right) .
$$

It is now easy to obtain that

$$
\varepsilon_{n}^{(1)}(C)<\varepsilon_{n}^{(\infty)}(C)=\frac{8}{x\left(1-\{\alpha(x)\}^{2}\right)}\{\alpha(x)\}^{n+2} .
$$

Following the procedure of Section 4 of [4], we obtain that

$$
\gamma_{n}(t)=\frac{1}{1-t}\left\{1-U_{n+1}\left(\frac{2 t}{x}-1\right) / U_{n+1}\left(\frac{2}{x}-1\right)\right\},
$$

where $U_{n}(v)$ is the Čebyšev polynomial of the second kind of degree $n$. Using this expression for $\gamma_{n}(t)$ and $t \beta_{n}(t)=\gamma_{n}(0)-(1-t) \gamma_{n}(t)$, we obtain that the entries of the corresponding matrix $B^{(1)}$ are given by

$$
\begin{aligned}
b_{n, k}^{(1)} & =\frac{(-1)^{n+k} 4^{k+1}}{x^{k+1} U_{n+1}(2 / x-1)}\left(\begin{array}{c}
n+k+3 \\
n-k
\end{array}\right) & & (0 \leqslant k \leqslant n) \\
& =0 & & (n>k) .
\end{aligned}
$$

The row sum is now $\gamma_{n}(0)=1-U_{n+1}(-1) / U_{n+1}(2 / x-1)$. From

$$
T_{n}(u)=\frac{1}{2}\left\{\left(u+\sqrt{u^{2}-1}\right)^{n}+\left(u-\sqrt{u^{2}-1}\right)^{n}\right\} \text { and } n U_{n-1}(u)=T_{n}^{\prime}(u),
$$

we obtain that

$$
U_{n}(u)=\frac{1}{2 \sqrt{u^{2}-1}}\left\{\left(u+\sqrt{u^{2}-1}\right)^{n+1}-\left(u-\sqrt{u^{2}-1}\right)^{n+1}\right\} .
$$


Thus

$$
U_{n+1}\left(\frac{2}{x}-1\right)=\frac{x}{4 \sqrt{1-x}}\left(\{\alpha(x)\}^{-n-2}-\{\alpha(x)\}^{n+2}\right) .
$$

It now follows that $\sum_{k=0}^{n} b_{n, k}^{(1)} \rightarrow 1$ as $n \rightarrow \infty$. It is also easy to obtain that $\lim _{n \rightarrow \infty} b_{n, k}^{(1)}=0$ for each $k$ and for each $x$. A procedure similar to that in Section 2 above shows that $\lim _{n \rightarrow \infty} \sum_{k=0}^{n}\left|b_{n, k}^{(1)}\right|=+\infty$, so that the matrix $B^{(1)}$ is not regular.

4. Numerical Considerations. Smith and Ford [5], [6] have done considerable numerical investigations to compare various accelerators. In particular, in Section 7 of [5], they reported on two series which are members of the class considered in this paper. See Tables 7.2 and 7.3 of [5]. The corresponding moment sequences $\left\{a_{n}\right\}$ are given by

$$
a_{n}=(0.5)^{n}+1 \text { and } a_{n}=1 /(n+1)
$$

with $x=0.8$.

With all the convergence accelerators tested by Smith and Ford, there are no general results giving the errors in the approximations. In the work of this paper and in [2], [3], and [4], all the errors are known, and the accuracy of the approximations is guaranteed.

We also point out that the matrices involved are very "nice". In the $L^{\infty}$ case we have rational functions and $\sqrt{1-x}$. In the $L^{1}$ case, we have rational functions alone.

Department of Mathematics

University of Western Ontario

London, Ontario, Canada N6A 5B9

1. N. I. ACHIESER, Theory of Approximation (C. J. Hyman, Transl.), Ungar, New York, 1956.

2. H. Fiedler \& W. B. JURKAT, "On best approximations of alternating series," J. Approx. Theory, v. 34,1982 , pp. $423-424$.

3. W. B. JuRKat \& B. L. R. Shawyer, “Best approximations of alternating series,” J. Approx. Theory, v. 34, 1982, pp. 397-422.

4. B. L. R. SHAWYer, "Best approximation of alternating power series," J. Math. Anal. Appl. (To appear.)

5. D. A. SMITH \& W. F. FORD, “Acceleration of linear and logarithmic convergence," SIAM J. Numer. Anal., v. 16, 1979, pp. 223-240.

6. D. A. SMith \& W. F. Ford, "Numerical comparisons of nonlinear convergence accelerators," Math. Comp., v. 38, 1982, pp. 481-499. 